\title{
In this issue: small B cell lymphomas, more than just a size
}

\author{
Leticia Quintanilla-Martinez $^{1} \cdot$ Steve H Swerdlow $^{1} \cdot$ Elias Campo $^{1}$
}

Published online: 17 February 2016

(C) Springer-Verlag Berlin Heidelberg 2016

This month's issue continues with another mini-series: the first two of four reviews on "small B-cell lymphomas in light of current technology", of which the remaining two will be published in next month's issue. These review papers resulted from the 2014 workshop of the European Association for Haematopathology/Society of Hematopathology held in Istanbul, Turkey.

Although mature B-cell lymphomas composed mainly of small lymphocytes are often referred to as "low-grade" B-cell lymphomas, the 2008 WHO classification of tumors of hematopoietic and lymphoid tissues intentionally does not divide lymphomas by grade and, because some are not necessarily indolent, more recently they are instead referred to as "small" B-cell lymphomas (SBL). They include chronic lymphocytic leukemia/small lymphocytic lymphoma (CLL/SLL), follicular lymphoma (FL), nodal marginal zone lymphoma (MZL), MALT lymphoma, hairy cell leukemia, lymphoplasmacytic lymphoma (LPL), and mantle cell lymphoma (MCL). Much has been learned about the SBL after the 2008 WHO classification and monograph was published, as a consequence of new and improved techniques used for standard diagnosis, translational research and more basic investigations. We have witnessed a growing list of genetic aberrations that are vari-

Leticia Quintanilla-Martinez

Leticia.quintanilla-fend@med.uni-tuebingen.de

Institute of Pathology and Neuropathology, Eberhard Karls University of Tübingen and Comprehensive Cancer Center, University Hospital Tübingen, , Liebermeisterstrasse 8, 72076 Tübingen, Germany ably present in SBL and that are useful either for diagnosis or for understanding the pathogenesis of these lymphomas. The identification of the recurrent MYD88 L265P mutation in $>90 \%$ of LPL is helping to delineate the morphological spectrum of this disease in extramedullary tissues. The recently described IRF4/MUM1 gene translocation in B-cell lymphomas is becoming the defining genetic alteration of a new entity affecting mostly children and young adults. Some discoveries found using molecular techniques, such as gene expression profiling, have been translated into immunohistochemical stains. These are now used in our daily diagnostic practice, including anti-SOX11 and LEF1 antibodies, used to help in the diagnosis of MCL and CLL, respectively. Nevertheless, despite all these new technologies, some old challenges persist. The border between some of the SBL remains ill-defined, especially in cases with plasmacytic differentiation. Transformation from an underlying indolent lymphoma to an aggressive lymphoma, mostly diffuse large B-cell lymphoma (DLBL), continues to be a diagnostic and clinical problem. The underlying mechanisms responsible for transformation are starting to be elucidated but prediction of the risk of transformation remains elusive. The articles within this series review the most common SBL with the aim of highlighting new molecular mechanisms, diagnostic challenges, unusual variants, useful criteria, new terminology and how to incorporate new technology into our routine diagnosis.

The reviews in this series include the following:

- The heterogeneity of follicular lymphomas: from early development to transformation.

- Indolent lymphomas in the paediatric population: follicular lymphoma, marginal zone lymphoma, IRF4/MUM1+ lymphomas, and chronic lymphocytic leukaemia.

- Mantle cell lymphoma: a spectrum from indolent to aggressive disease. 
- The many faces of small B-cell lymphomas with plasmacytic differentiation and the contribution of MYD 88 testing.

Xerri et al. (10.1007/s00428-015-1864-y) discuss heterogeneity of follicular lymphoma (FL), a lymphoma derived from germinal center (GC) B-cells that in $85 \%$ of the cases carry the $\mathrm{t}(14 ; 18)$ chromosomal alteration with overexpression of the anti-apoptotic protein BCL2. They address the issues of clinical and morphological heterogeneity of FL, as well as the complexity of cytogenetic and molecular abnormalities observed in cases that lack the characteristic BCL2 translocation that might result from different genetic mechanisms operating within the GC. They discuss the complexity of FL transformation into different entities, such as lymphoblastic lymphoma or histiocytic sarcoma, which is attributable to different modalities of evolution of the precursor or founder cell clone or to the presence of several subclones is discussed.

Quintanilla-Martinez et al. (DOI 10.1007/s00428-015$1855-z)$ review indolent lymphomas in de paediatric population. The vast majority of B-cell NHLs in the paediatric age are aggressive lymphomas including Burkitt lymphoma, B lymphoblastic lymphoma and diffuse large B-cell lymphoma. SBL in children and young adults are very uncommon but the two more frequent are FL and nodal marginal zone lymphoma (NMZL). Paediatric type FL differs clinically, morphologically and genetically from the adult counterpart. The review highlights the main characteristics of paediatric type FL and paediatric NMZL and recognizes the overlapping features. Nevertheless, morphologic and immunophenotypic criteria are helpful for the differential diagnosis and these are presented. Both paediatric type FL and pediatric NMZL are indolent processes that should be distinguished from atypical lymphoid hyperplasia of the tonsils and lymph nodes. The newly recognized entity of IRF4/MUM1+ lymphomas mainly involving Waldeyer's ring in children and young adults is presented and its differential diagnosis with paediatric type FL is emphasized. The importance of recognizing IRF4/MUM1+ FL/DLBCL as a distinct entity, which usually requires therapy, is underscored.

The reviews presented in this series (in this issue or in the next) show how new technologies have increased our understanding of the biological diversity of SBL. The morphological and immunophenotypical variation within the different SBL subtypes should be recognized so that patients can be treated appropriately. As new markers and genetic alterations are identified, the need to standardize molecular testing for accurate diagnosis and/or prognostication in daily practice is required. The reviews also highlight the relevance of systematic expert review of unusual cases, exemplified in the traditional annual Workshops jointly organized by the European Association for Haematopathology and Society for Hematopathology. Such activities allow the collection and study of a large number of cases including unusual and less well known variants that can be investigated and discussed by participants from around the world offering a unique possibility to generate new knowledge and refine diagnostic criteria. At the same time such workshops raise new questions and perspectives that foster future investigations.

The cover image was taken from the Quintanilla-Martinez et al. paper (fig. $4 b)$.

\section{Leticia Quintanilla-Martinez}

Associate Editor Virchows Archiv, guest Editor of the miniseries

Leticia.quintanilla-fend@med.uni-tuebingen.de

Steve H Swerdlow

Elias Campo 